\title{
Exploring challenges of RCTs in the emergency and critical care setting
}

\author{
Paul Mouncey*, Sarah Power, David Harrison, Sheila Harvey, Kathryn Rowan \\ From 3rd International Clinical Trials Methodology Conference \\ Glasgow, UK. 16-17 November 2015
}

\section{Introduction}

Approximately $50 \%$ of randomised controlled trials (RCTs) fail to recruit to time and target. RCTs in the emergency and critical care settings pose additional challenges including time of presentation of potentially eligible patients and the time required to formally recruit patients.

\section{Objectives}

To describe the screening and recruitment patterns in RCTs in an emergency/critical care setting in the NHS and to explore the impact that time taken to consent patients may have on the delivery of an early intervention.

\section{Methods}

We recently completed two large multicentre RCTs in the emergency and critical care setting. Both evaluated delivery of an early intervention - early, goal-directed therapy (a resuscitation protocol) ( $\mathrm{n}=1260$ patients; 56 sites) and early nutritional support ( $\mathrm{n}=2400$ patients; 33 sites). As well as collecting data for the evaluation, we also collected data around screening, recruitment and timing.

\section{Results}

In both trials, due to the setting, recruitment rates were lower - with eligible patients missed - at nights and at weekends with an absence of study "delegated" staff available often cited as the reason. In both trials, $81 \%$ of patients were recruited Monday to Friday 08:00 to 19:59. In the trial on resuscitation, time taken to formally recruit patients impacted on the early nature of the intervention (meeting inclusion criteria to randomisation was on average 1.1 hours).

ICNARC Clinical Trials Unit, London, UK 